(REVIEW ARTICLE)

\title{
Intellectual fashion forecasting simulations and application
}

\author{
Bhuiyan Md Jashim Uddin ${ }^{1}$, Kamran Muhammad 2, ${ }^{*}$, Uddin Md Nazim ${ }^{3}$ and Razzaq Abdul ${ }^{1}$ \\ ${ }^{1}$ Management Science and Engineering, Kunming University of Science and Technology, Kunming, PR China. \\ 2 Department of Economics, Government College University, Faisalabad, Pakistan. \\ ${ }^{3}$ Department of Management, Mohammadpur Kendriya College, Dhaka, Bangladesh.
}

Publication history: Received on 24 June 2020; revised on 13 July 2020; accepted on 15 July 2020

Article DOI: https://doi.org/10.30574/wjarr.2020.7.1.0231

\begin{abstract}
For over 40 years Boeing has published an annual forecast for the growth of air travel worldwide. Current Market Outlook has become a standard reference for planners in government and industry. Major methodological changes in the forecast occurred in mid of 1990. These were a result of rethinking the data, methods, and models. It turns out standard approaches over-emphasized the effect of GDP. On the other hand, statistics found a convincing and cogent link between trade and travel, even though the data appeared entirely random. Finally, a startlingly simple approach established the hitherto elusive link between travel and service quality. This talk is a light-hearted review of the things we did wrong and fixed, and the things we are still worried about. It provides a bittersweet contrast between the ways of industry and academia. In this study we discussed Identifying and tracking fashion trends, apply knowledge of fashion forecasting to understand how trends evolve and are interpreted by industry. As a bonus we introduce Occam's Toothbrush an entirely new, hopelessly backwards, and entirely un-publishable way industry does hypothesis testing, but doesn't realize it.
\end{abstract}

Keywords: Forecast; Fashion; Trends; Approach; Standard; Market; Backwards; Industry

\section{Introduction}

Fashion is a fascinating domain for computer vision. Not only does it offer a challenging test bed for fundamental vision problems human body parsing, cross domain image matching, and recognition but it also inspires new problems that can drive a research agenda, such as modeling visual compatibility, interactive fine-grained retrieval or reading social cues from what people choose to wear $[1,2]$. Traditionally, fashion sales forecasting is accomplished by the statistical methods. In fact, a lot of statistical methods have been used for sales forecasting, which include linear regression, moving average, weighted average, exponential smoothing (used when a trend is present but not linear), exponential smoothing with trend, double exponential smoothing, Bayesian analysis, and so forth. Statistical time series analysis tools such as ARIMA and SARIMA are widely employed in sales forecasting [3]. Since these methods have a closed form expression for forecasting, it is simple and easy to implement and the results can be computed very quickly. In the literature, [4] apply a Bayesian approach to explore forecasting for a mail order company which sells ladies dresses. After that, [5] use item classification to examine the accuracy of sales forecasting for new items. They find that a larger number of item families and pertinent classification criteria are required in the respective forecasting procedure in order to achieve an improved forecasting precision. They conclude that product family and aggregated forecasting are more accurate than the individual item's forecasting. Recently, [6] also consider the forecasting problem based on a case study of a mail order apparel company. They propose a "top-flop" classification method and argue that it performs better than other methods. Furthermore, they find that the expert judgment methods outperform the advance demand information method for a small group of products. Another recent work [7] examines the applicability of a Bayesian forecasting model for fashion demand forecasting. It is found that the proposed hierarchical Bayesian approach yields superior quantitative results compared to many other methods.

\footnotetext{
* Corresponding author: Kamran Muhammad
} 


\section{Forecasting Methodology}

Most people view the world as consisting of a large number of alternatives. Futures research evolved as a way of examining the alternative futures and identifying the most probable. Forecasting is designed to help decision making and planning in the present. Forecasts empower people because their use implies that we can modify variables now to alter (or be prepared for) the future. A prediction is an invitation to introduce change into a system.

There are several assumptions about forecasting:

- There is no way to state what the future will be with complete certainty. Regardless of the methods that we use there will always be an element of uncertainty until the forecast horizon has come to pass.

- There will always be blind spots in forecasts. We cannot, for example, forecast completely new technologies for which there are no existing paradigms.

- Providing forecasts to policy-makers will help them formulate social policy. The new social policy, in turn, will affect the future, thus changing the accuracy of the forecast.

Many scholars have proposed a variety of ways to categorize forecasting methodologies. The following classification is a modification of the schema developed by Gordon over two decades ago.

Genius forecasting: This method is based on a combination of intuition, insight, and luck. Psychics and crystal ball readers are the most extreme case of genius forecasting. Their forecasts are based exclusively on intuition. Science fiction writers have sometimes described new technologies with uncanny accuracy.

There are many examples where men and women have been remarkable successful at predicting the future. There are also many examples of wrong forecasts. The weakness in genius forecasting is that it's impossible to recognize a good forecast until the forecast has come to pass. Some psychic individuals are capable of producing consistently accurate forecasts. Mainstream science generally ignores this fact because the implications are simply too difficult to accept. Our current understanding of reality is not adequate to explain these phenomena. Trend extrapolation - These methods examine trends and cycles in historical data, and then use mathematical techniques to extrapolate to the future. The assumption of all these techniques is that the forces responsible for creating the past will continue to operate in the future. This is often a valid assumption when forecasting short term horizons, but it falls short when creating medium and long term forecasts. The further out we attempt to forecast, the less certain we become of the forecast.

The stability of the environment is the key factor in determining whether trend extrapolation is an appropriate forecasting model. The concept of "developmental inertia" embodies the idea that some items are more easily changed than others. Clothing styles is an example of an area that contains little inertia. It is difficult to produce reliable mathematical forecasts for clothing. Energy consumption, on the other hand, contains substantial inertia and mathematical techniques work well. The developmental inertia of new industries or new technology cannot be determined because there is not yet a history of data to draw from. There are many mathematical models for forecasting trends and cycles. Choosing an appropriate model for a particular forecasting application depends on the historical data. The study of the historical data is called exploratory data analysis. Its purpose is to identify the trends and cycles in the data so that appropriate model can be chosen. The most common mathematical models involve various forms of weighted smoothing methods. Another type of model is known as decomposition. This technique mathematically separates the historical data into trend, seasonal and random components. A process known as a "turning point analysis" is used to produce forecasts. ARIMA models such as adaptive filtering and Box-Jenkins analysis constitute a third class of mathematical model, while simple linear regression and curve fitting is a fourth. 


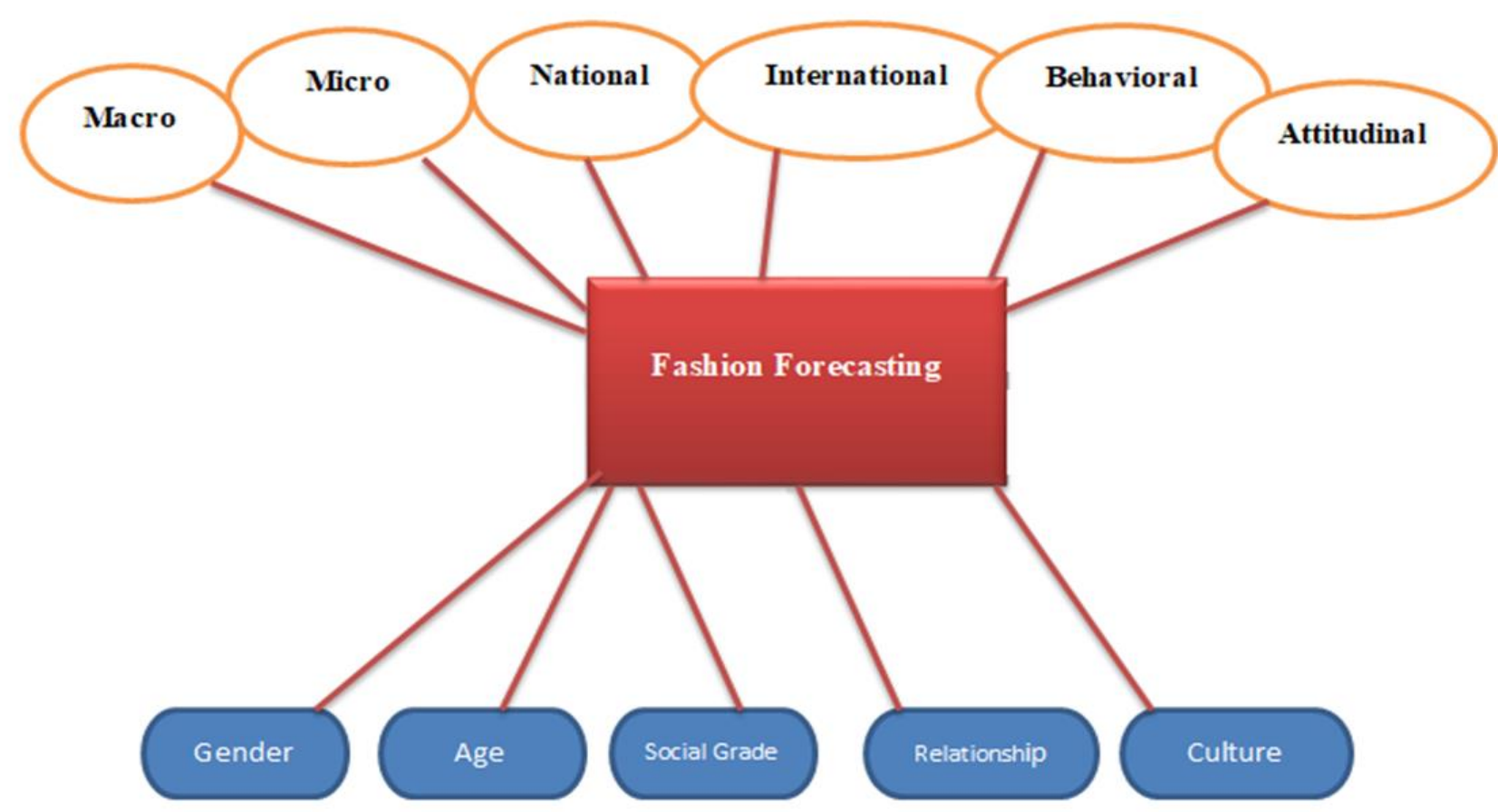

Figure 1 Associations in fashion forecasting

The common feature of these mathematical models is that historical data is the only criteria for producing a forecast. One might think then, that if two people use the same model on the same data that the forecasts will also be the same, but this is not necessarily the case. Mathematical models involve smoothing constants, coefficients and other parameters that must decide by the forecaster. To a large degree, the choice of these parameters determines the forecast. It is vogue today to diminish the value of mathematical extrapolation. Makridakis (one of the gurus of quantitative forecasting) correctly points out that judgmental forecasting is superior to mathematical models; however, there are many forecasting applications where computer generated forecasts are more feasible. For example, large manufacturing companies often forecast inventory levels for thousands of items each month. It would simply not be feasible to use judgmental forecasting in this kind of application.

\subsection{Consensus methods}

Forecasting complex systems often involves seeking expert opinions from more than one person. Each is an expert in his own discipline, and it is through the synthesis of these opinions that a final forecast is obtained. One method of arriving at a consensus forecast would be to put all the experts in a room and let them "argue it out". This method falls short because the situation is often controlled by those individuals that have the best group interaction and persuasion skills. A better method is known as the Delphi technique. This method seeks to rectify the problems of face-to-face confrontation in the group, so the responses and respondents remain anonymous. The classical technique proceeds in well-defined sequence. In the first round, the participants are asked to write their predictions. Their responses are collated and a copy is given to each of the participants. The participants are asked to comment on extreme views and to defend or modify their original opinion based on what the other participants have written. Again, the answers are collated and fed back to the participants. In the final round, participants are asked to reassess their original opinion in view of those presented by other participants.

The Delphi method general produces a rapid narrowing of opinions. It provides more accurate forecasts than group discussions. Furthermore, a face-to-face discussion following the application of the Delphi method generally degrades accuracy. Simulation methods - Simulation methods involve using analogs to model complex systems. These analogs can take on several forms. A mechanical analog might be a wind tunnel for modeling aircraft performance. An equation to predict an economic measure would be a mathematical analog. A metaphorical analog could involve using the growth of a bacteria colony to describe human population growth. Game analogs are used where the interactions of the players are symbolic of social interactions. 
Mathematical analogs are of particular importance to futures research. They have been extremely successful in many forecasting applications, especially in the physical sciences. In the social sciences however, their accuracy is somewhat diminished. The extraordinary complexity of social systems makes it difficult to include all the relevant factors in any model. Clarke reminds us of a potential danger in our reliance on mathematical models. As he points out, these techniques often begin with an initial set of assumptions, and if these are incorrect, then the forecasts will reflect and amplify these errors. One of the most common mathematical analogs in societal growth is the S-curve. The model is based on the concept of the logistic or normal probability distribution. All processes experience exponential growth and reach an upper asymptotic limit. Modis has hypothesized that chaos like states exist at the beginning and end of the Scurve. The disadvantage of this S-curve model is that it is difficult to know at any point in time where you currently are on the curve, or how close you are to the asymptotic limit. The advantage of the model is that it forces planners to take a long-term look at the future.

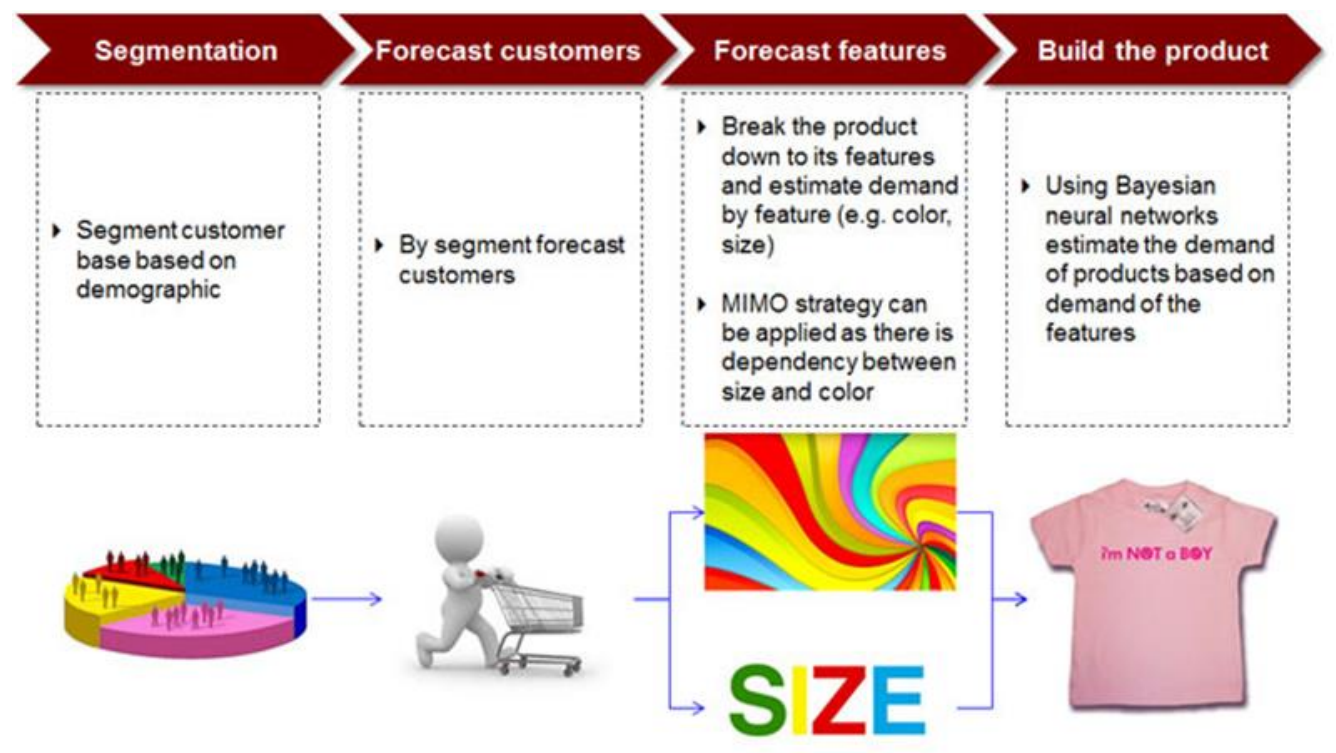

Figure 2 Schematic view of secondary research for the forecasting fashion

Another common mathematical analog involves the use of multivariate statistical techniques. These techniques are used to model complex systems involving relationships between two or more variables. Multiple regression analysis is the most common technique. Unlike trend extrapolation models, which only look at the history of the variable being forecast, multiple regression models look at the relationship between the variable being forecast and two or more other variables. Multiple regressions are the mathematical analog of a systems approach, and it has become the primary forecasting tool of economists and social scientists. The object of multiple regressions is to be able to understand how a group of variables (working in unison) affect another variable. The multiple regression problem of collinearity mirrors the practical problems of a systems approach. Paradoxically, strong correlations between predictor variables create unstable forecasts, where a slight change in one variable can have dramatic impact on another variable. In a multiple regression (and systems) approach, as the relationships between the components of the system increase, our ability to predict any given component decreases.

Gaming analogs are also important to futures research. Gaming involves the creation of an artificial environment or situation. Players (either real people or computer players) are asked to act out an assigned role. The "role" is essentially a set of rules that is used during interactions with other players. While gaming has not yet been proven as a forecasting technique, it does serve two important functions. First, by the act of designing the game, researchers learn to define the parameters of the system they are studying. Second, it teaches researchers about the relationships between the components of the system. Cross-impact matrix method - Relationships often exist between events and developments that are not revealed by univariate forecasting techniques. The cross-impact matrix method recognizes that the occurrence of an event can, in turn, affect the likelihoods of other events. Probabilities are assigned to reflect the likelihood of an event in the presence and absence of other events. The resultant inter-correlational structure can be used to examine the relationships of the components to each other, and within the overall system. The advantage of this technique is that it forces forecasters and policy-makers to look at the relationships between system components, rather than viewing any variable as working independently of the others. 


\subsection{Scenario}

The scenario is a narrative forecast that describes a potential course of events. Like the cross-impact matrix method, it recognizes the interrelationships of system components. The scenario describes the impact on the other components and the system as a whole. It is a "script" for defining the particulars of an uncertain future. Scenarios consider events such as new technology, population shifts, and changing consumer preferences. Scenarios are written as long-term predictions of the future. A most likely scenario is usually written, along with at least one optimistic and one pessimistic scenario. The primary purpose of a scenario is to provoke thinking of decision makers who can then posture themselves for the fulfillment of the scenario(s). The three scenarios force decision makers to ask: 1 . can we survive the pessimistic scenario, 2. are we happy with the most likely scenario, and 3. are we ready to take advantage of the optimistic scenario? Decision trees - Decision trees originally evolved as graphical devices to help illustrate the structural relationships between alternative choices. These trees were originally presented as a series of yes/no (dichotomous) choices. As our understanding of feedback loops improved, decision trees became more complex. Their structure became the foundation of computer flow charts.

Computer technology has made it possible create very complex decision trees consisting of many subsystems and feedback loops. Decisions are no longer limited to dichotomies; they now involve assigning probabilities to the likelihood of any particular path. Decision theory is based on the concept that an expected value of a discrete variable can be calculated as the average value for that variable. The expected value is especially useful for decision makers because it represents the most likely value based on the probabilities of the distribution function. The application of Bayes' theorem enables the modification of initial probability estimates, so the decision tree becomes refined as new evidence is introduced. Utility theory is often used in conjunction with decision theory to improve the decision making process. It recognizes that dollar amounts are not the only consideration in the decision process. Other factors, such as risk, are also considered.

\subsection{Combining Forecasts}

It seems clear that no forecasting technique is appropriate for all situations. There is substantial evidence to demonstrate that combining individual forecasts produces gains in forecasting accuracy. There is also evidence that adding quantitative forecasts to qualitative forecasts reduces accuracy. Research has not yet revealed the conditions or methods for the optimal combinations of forecasts. Judgmental forecasting usually involves combining forecasts from more than one source. Informed forecasting begins with a set of key assumptions and then uses a combination of historical data and expert opinions. Involved forecasting seeks the opinions of all those directly affected by the forecast (e.g., the sales force would be included in the forecasting process). These techniques generally produce higher quality forecasts than can be attained from a single source. Combining forecasts provides us with a way to compensate for deficiencies in a forecasting technique. By selecting complementary methods, the shortcomings of one technique can be offset by the advantages of another.

\section{Difficulties in Forecasting Technology}

Clarke describes our inability to forecast technological futures as a failure of nerve. When a major technological breakthrough does occur, it takes conviction and courage to accept the implications of the finding. Even when the truth is starring us in the face, we often have difficulty accepting its implications. Clark refers to this resistance to change as cowardice; however, it may be much deeper. Cognitive dissonance theory in psychology has helped us understand that resistance to change is a natural human characteristic. It is extremely difficult to venture beyond our latitudes of acceptance in forecasting new technologies.

Clarke states that knowledge can sometimes clog the wheels of imagination. He embodied this belief in his selfproclaimed law: "When a distinguished but elderly scientist states that something is possible, he is almost certainly right. When he states that something is impossible, he is very probably wrong. "Nearly all futurists describe the past as unchangeable, consisting as a collection of knowable facts. We generally perceive the existence of only one past. When two people give conflicting stories of the past, we tend to believe that one of them must be lying or mistaken. This widely accepted view of the past might not be correct. Historians often interject their own beliefs and biases when they write about the past. Facts become distorted and altered over time. It may be that past is a reflection of our current conceptual reference. In the most extreme viewpoint, the concept of time itself comes into question. The future, on the other hand, is filled will uncertainty. Facts give way to opinions. As de Jouvenel points out, the facts of the past provide the raw materials from which the mind makes estimates of the future. All forecasts are opinions of the future (some more carefully formulated than others). The act of making a forecast is the expression of an opinion. The future, as described by de Jouvenel, consists of a range of possible future phenomena or events. These futureless are those things that might happen. 


\section{Defining a Useful Forecast}

Science fiction novelist Frederik Pohl has suggested that the "only time a forecast has any real utility is when it is not totally reliable". He proposes a thought experiment where a Gypsy fortune teller predicts that we will be run over and killed when we leave the tea room. If we know that the Gypsy's predictions are one hundred percent accurate, then Pohl states that the fortune is useless, because we would be unable to alter the forecast. In other words, predictions only become useful when they are not completely reliable.

The apparent paradox created by Pohl's thought experiment is only a function of the particular situation. The paradox exists only when 1 . We want the future to be different than the prediction, and 2 . When we believe that there is no way for us to adapt to or affect the forthcoming changes. Pohl's thought experiment actually doesn't even meet that criteria, since one could present a convincing argument that it is more desirable to spend the rest of our lives confined to the comfort of a tea room than to leave and meet certain death. Obviously, our new life would be difficult to accept and adapt to, but it could be done. Prisoners do it all the time.

A forecast can be one hundred percent accurate and still be useful. For example, suppose our Gypsy had told us that after leaving her tea room we would safely return home. Again, since we know that her forecasts are completely accurate, we would receive emotional comfort from her predictions. In a more tangible example, suppose the prediction is that our manufacturing company will receive twice as many orders for widgets as we had anticipated. Since the forecast is one hundred percent accurate we would be wise to order more raw materials and increase our production staff to meet the coming demand.

Pohl is wrong. The goal of forecasting is to be as accurate as possible. In the case of business demand forecasting, it is naive to suggest that an accurate forecast is useless. On the contrary, a more accurate forecast enables us to plan the use our resources in a more ecological fashion. We can minimize waste by adapting to our expectations of the future.

It is sometimes useful in thought experiments to look at the situation from the opposite perspective. Suppose we know that our Gypsy is always wrong in her predictions. Her accuracy is guaranteed to be zero. Note that this is different than random forecasts, where she might hit the mark once in a while. The Gypsy sighs with relief and says that there is no fatal accident in store for us today. According to Pohl's reasoning, this should provide the most useful forecast because it has the least accuracy. It's obvious, though, that this fortune is as useless as the one where she is completely accurate. Leaving the Gypsy's tea-room is not something we would want to do.

If we view accuracy as a continuum, it may be that the antonym of accuracy is randomness (instead of inaccuracy). In this case, Pohl's theory would suggest that random forecasts are more useful than accurate forecasts. In demand forecasting, the degree of over- and under-utilization of our resources is proportional to the difference between the observed and predicted values. Random forecasts are entirely unacceptable for this type of application.

Pohl's thought experiment is very important because it forces us to look at the theoretical foundations of forecasting. First, Pohl's experiment may not be valid because it violates a basic assumption of forecasting (i.e., we cannot predict the future with one hundred percent accuracy). Second, the usefulness of a forecast does not always seem to be related to its accuracy. Both extremes (completely accurate and completely inaccurate) can produce useful or useless forecasts.

The usefulness of a forecast is not something that lends itself readily to quantification along any specific dimension (such as accuracy). It involves complex relationships between many things, including the type of information being forecast, our confidence in the accuracy of the forecast, the magnitude of our dissatisfaction with the forecast, and the versatility of ways that we can adapt to or modify the forecast. In other words, the usefulness of a forecast is an application sensitive construct. Each forecasting situation must be evaluated individually regarding its usefulness. One of the first rules of doing research is to consider how the results will be used. It is important to consider who the readers of the final report will be during the initial planning stages of a project. It is wasteful to expend resources on research that has little or no use. The same rule applies to forecasting. We must strive to develop forecasts that are of maximum usefulness to planners. This means that each situation must be evaluated individually as to the methodology and type of forecasts that are most appropriate to the particular application.

\section{Do Forecasts Create the Future?}

A paradox exists in preparing a forecast. If a forecast results in an adaptive change, then the accuracy of the forecast might be modified by that change. Suppose the forecast is that our business will experience a ten percent drop in sales 
next month. We adapt by increasing our promotion effort to compensate for the predicted loss. This action, in turn, could affect our sales, thus changing the accuracy of the original forecast. Many futurists (de Jouvenel, Dublin, Pohl, and others) have expressed the idea that the way we contemplate the future is an expression of our desire to create that future. Physicist Dennis Gabor, discoverer of holography, claimed that the future is invented, not predicted. The implication is that the future is an expression of our present thoughts. The idea that we create our own reality is not a new concept. It is easy to imagine how thoughts might translate into actions that affect the future.

Biblical records speak of faith as the force that could move mountains. Recent research in quantum mechanics suggests that this may be more than just a philosophical concept. At a quantum level, matter itself might simply be a manifestation of thought. Electrons and other subatomic particles seem to exist only when physicists are looking for them, otherwise, they exist only as energy.

An incredible discovery was made at the University of Paris in 1982. A team of researchers lead by Alain Aspect found that under certain conditions, electrons could instantaneously communicate with each other across long distances. The results of this experiment have been confirmed by many other researchers, although the implications are exceedingly hard to accept. Three explanations are possible: 1 . information can be transferred at speeds exceeding the speed of light 2. The passage of time is an illusion 3. The distance between the electrons is an illusion. All three explanations rock our perception of reality. David Bohm has explained Aspect's experiment by hypothesizing a holographic universe in which reality is essentially a projection of some deeper dimension that we are not able to comprehend. Instantaneous communication is possible because the distance between the particles is an illusion. Neurophysiologist Karl Pribram has also theorized about the holographic nature of reality. His theory is based on a study of the way that the brain recalls memory patterns, but the implications are the same. Reality is a phantasm.

\section{If reality is an illusion, then the future is also an illusion}

The phenomena of being able to see the future is known as precognition. Most people believe that (to some degree) they can predict the future. Fortune-tellers, however, believe they can view the future. There is a major difference. We predict the future based on knowledge, intuition and logic. Precognitive persons claim to see the future. Knowledge and logic are not involved. Throughout history, there have been many reports of gifted psychics with precognitive powers. Through some unknown mechanism, these people are able predict things that will happen in the future. If we admit that even a single person in history has possessed this capability, then we must accept the fact that our concept of reality needs dramatic alteration. Time itself may not exist as we currently perceive it. Forecasting may be a method of creating illusions.

Forecasting can, and often does, contribute to the creation of the future, but it is clear that other factors are also operating. A holographic theory would stress the interconnectedness of all elements in the system. At some level, everything contributes to the creation of the future. The degree to which a forecast can shape the future (or our perception of the future) has yet to be determined experimentally and experientially. Sometimes forecasts become part of a creative process, and sometimes they don't. When two people make mutually exclusive forecasts, both of them cannot be true. At least one forecast is wrong. Does one person's forecast create the future, and the other does not? The mechanisms involved in the construction of the future are not well understood on an individual or social level. Modis believes that the media provides the mechanism by which social forecasts take on a creative context. In this theory, extensive media coverage acts as a resonating cavity for public opinion, and creates a "cultural epidemic" that modifies social behavior. Dublin points out that the "future has become so integral to the fabric of modern consciousness that few people feel compelled to question it..." Because of the power of a prediction to affect the future, he goes on to state that prophesy is usually a self-interest quest for power.

\section{The Ethics of Forecasting}

Are predictions of the future a form of propaganda, designed to evoke a particular set of behaviors? Dublin states that the desire for control is implicit in all forecasts. Decisions made today are based on forecasts, which may or may not come to pass. The forecast is a way to control today's decisions. Dublin is correct. The purpose of forecasting is to control the present. In fact, one of the assumptions of forecasting is that the forecasts will be used by policy-makers to make decisions. It is therefore important to discuss the ethics of forecasting. Since forecasts can and often do take on a creative role, what right do we have to make forecasts that involve other people's futures? Nearly everyone would agree that we have the right to create our own future. Goal setting is a form of personal forecasting. It is one way to organize and invent our personal future. Each person has the right to create their own future. On the other hand, a social forecast might alter the course of an entire society. Such power can only be accompanied by equivalent responsibility. 
There are no clear rules involving the ethics of forecasting. In Future Shock, Toffler discussed the importance of value impact forecasting, the idea that social forecasting must involve physical, cultural and societal values. It is doubtful that forecasters can leave their own personal biases out of the forecasting process. Even the most mathematically rigorous techniques involve judgmental inputs that can dramatically alter the forecast. Many futurists have pointed out our obligation to create socially desirable futures. Unfortunately, a socially desirable future for one person might be another person's nightmare. For example, modern ecological theory says that we should think of our planet in terms of sustainable futures. The finite supply of natural resources forces us to reconsider the desirability of unlimited growth. An optimistic forecast is that we achieve and maintain an ecologically balanced future. That same forecast, the idea of zero growth, is a catastrophic nightmare for the corporate and financial institutions of the free world. Our Keynesian system of profit depends on continual growth for the well-being of individuals, groups, and institutions. Desirable futures are a subjective concept. It can only be understood relative to other information. The ethics of forecasting certainly involves the obligation to create desirable futures for the persons that might be affected by the forecast. If a goal of forecasting is to create desirable futures, then the forecaster must ask the ethical question of desirable for whom? To embrace the idea of liberty is to recognize that each person has the right to create their own future. Forecasters can promote libertarian beliefs by empowering people that might be affected by the forecast. Involving these people in the forecasting process, gives them the power to become co-creators in their futures.

\section{Forthcoming Recommendation}

- For fashion retail sales forecasting, regarding the data source, there are three kinds of data, namely, the time series data, cross-section data, and panel data. The time series data, which is collected over discrete intervals of time, is widely used in fashion forecasting and the methods applied to time-series data are also well developed. Cross-section data is collected over sample units in a particular time period and panel data follows individual micro units over time. These two kinds of data are not yet fully used for fashion sales forecasting. Recently, a forecasting method using the panel data is developed in and it will be an interesting future research direction to explore the use of these different types of data for fashion sales forecasting.

- Color is one critical element in fashion and it is highly related to the inventory and production planning of fashion apparel products. However, from the reviewed literature, only very few prior studies have examined color forecasting. Thus, more studies on this topic can be conducted. In addition, on a related area, no prior study has examined how fashion pattern design and other design factors affect demand and the respective sales forecasting mechanism. It is another interesting topic for further studies.

- In fashion retail system, the sales of the apparel product are strongly influenced by the calendar factor, for example, holiday. It can be observed easily that the sales in National day's holidays in Hong Kong and Black Friday holidays in the USA will go up very quickly and highly. On one hand, the demands on these specific dates are much more volatile and difficult to predict. On the other hand, the revenue that can be generated during these periods of time can be huge. As a consequence, how to precisely forecast the demand during special dates/events becomes crucial to fashion retailors. This becomes another topic open for future research.

\section{Conclusion}

So, during doing this report I face lots of issue what will help me to build up a new business. Spain is best place for doing business, because there were so many opportunities on this country. By this report research on the particular country Spain there some strong point of opportunities like, tourist, culture conscious, fashion conscious and lots of international football match happened there. So, on that particular country you can easily setup a new business. It is important for any business start that you know all about it. In the fashion industry, predicting trends, due to its complexity, are frequently compared to weather forecasting: sometimes you get it right and sometimes you get it wrong. In this work, we show that using our vision-based fashion forecasting model we get it right more often than not. We propose a model that discovers fine-grained visual styles from large scale fashion data in an unsupervised manner. Our model identifies unique style signatures and provides a semantic description for each based on key visual attributes. Furthermore, based on user consumption behavior, our model predicts the future popularity of the styles, and reveals their life cycle and status (e.g. in- or out of fashion). We show that vision is essential for reliable forecasts, outperforming textual-based representations. Finally, fashion is not restricted to apparel; it is present in accessories, automobiles, and even house furniture. Our model is generic enough to be employed in different domains where a notion of visual style is present. 


\section{Compliance with ethical standards}

\section{Acknowledgments}

Authors delightfully acknowledge the Kunming University of Science and Technology, Kunming, China for supporting this study. Authors also acknowledge the sincere efforts of Dr. Ghulam Murtaza (Kunming University of Science and Technology, Kunming, China) for improving the final draft of the manuscript.

\section{Disclosure of conflict of interest}

All the authors have no conflict of interest.

\section{References}

[1] Chen Q, Huang J, Feris R, Brown LM, Dong J and Yan S. (2015). Deep Domain Adaptation for Describing People Based on Fine-Grained Clothing Attributes. In CVPR.

[2] Liu S, Feng J, Song Z, Zhang T, Lu H, Xu C and Yan S. (2012). Hi, Magic Closet, Tell Me What to Wear. In ACM Multimedia.

[3] Box GEP, Jenkins GM and Reinsel GC. (2008). Time Series Analysis: Forecasting and Control, Wiley Series in Probability and Statistics, John Wiley \& Sons, Hoboken, NJ, USA, 4th edition.

[4] Green M and Harrison PJ. (1973). Fashion forecasting for a mail order company using a bayesian approach. Operational Research Quarterly, 2, 193-205.

[5] Thomassey S, Happiette M and Castelain JM. (2003). Mean-term textile sales forecasting using families and items classification. Studies in Informatics and Control, 12, 41-52.

[6] Mostard J, Teunter R and De Koster R. (2011). Forecasting demand for single-period products: a case study in the apparel industry. European Journal of Operational Research, 211, 139-147.

[7] Yelland PM and Dong XJ. (2013). forecasting demand for fashion goods: a hierarchical Bayesian approach. In Handbook on Intelligent Fashion Forecasting Systems.

\section{How to cite this article}

Bhuiyan MU, Kamran M, Uddin MN and Razzaq A. (2020). Intellectual fashion forecasting simulations and application. World Journal of Advanced Research and Reviews, 7(1), 133-141. 